\title{
ON THE CONDITIONAL REGULARITY OF THE NAVIER-STOKES AND RELATED EQUATIONS
}

\author{
DONGHO CHAE \\ Department of Mathematics \\ Sungkyunkwan University \\ Suwon 440-746, Korea \\ E-mail: chae@skku.edu
}

\begin{abstract}
We present regularity conditions for a solution to the 3D Navier-Stokes equations, the $3 \mathrm{D}$ Euler equations and the 2D quasigeostrophic equations, considering the vorticity directions together with the vorticity magnitude. It is found that the regularity of the vorticity direction fields is most naturally measured in terms of norms of the Triebel-Lizorkin type.
\end{abstract}

1. The generalized Navier-Stokes equations. In this section we are concerned with the following 'generalized' Navier-Stokes equations:

$$
\begin{gathered}
\frac{\partial v}{\partial t}+(v \cdot \nabla) v=-\nabla p-\nu \Lambda^{\alpha} v, \\
\operatorname{div} v=0, \\
v(x, 0)=v_{0}(x),
\end{gathered}
$$

where $v=\left(v^{1}, v^{2}, v^{3}\right), v^{j}=v^{j}(x, t), j=1,2,3$, is the velocity of the fluid flows, $p=p(x, t)$ is the scalar pressure, $v_{0}(x)$ is a given initial velocity field satisfying $\operatorname{div} v_{0}=0$, and $\nu>0$ is the viscosity constant. We write $\Lambda^{\alpha}=(-\Delta)^{\frac{\alpha}{2}}$. We denote the system (1)-(3) by $(N S)_{\alpha}$. The case $\alpha=2$ corresponds to the usual Navier-Stokes equations, which we denote simply by $(N S)$. Heuristically, the number $\alpha$ represents the 'strength of dissipation', and in this paper we are concerned with the case $0<\alpha \leq 2$. We note that the system $(N S)_{\alpha}$ was first considered by J.-L. Lions in [26], and the global regularity $\alpha \geq \frac{5}{2}$ is shown there. Taking curl of (1) we obtain the following vorticity equation:

$$
\frac{\partial \omega}{\partial t}+(v \cdot \nabla) \omega=(\omega \cdot \nabla) v-\nu \Lambda^{\alpha} \omega
$$

2000 Mathematics Subject Classification: 35Q35, 76B03, 76D05.

Key words and phrases: regularity condition, Navier-Stokes equations, Euler equations, quasigeostrophic equations.

This research was supported by KOSEF Grant no. R01-2005-000-10077-0. 
where the vorticity $\omega$ is defined by

$$
\omega=\operatorname{curl} v .
$$

The incompressibility condition (2) combined with (5) implies the Biot-Savart law,

$$
v(x, t)=-\frac{1}{4 \pi} \int_{\mathbb{R}^{3}} \frac{y \times \omega(x+y, t)}{|y|^{3}} d y
$$

for sufficiently rapidly decaying vorticity near infinity. After pioneering work by Leray ([25]) there are many comprehensive papers on the existence theory of the weak solution of the $(N S)$ equations, $[24,35,27]$. The regularity of this weak solution is known as one of the most challenging problems in the mathematical fluid mechanics. In this note we are concerned with the regularity condition of the weak solutions of $(N S)_{\alpha}$. The pioneering results for $(N S)$ in this direction are $[28,29,32,23]$, which state that if a weak solution $v(x, t)$ satisfies

$$
v \in L^{r}\left(0, T ; L^{p}\left(\mathbb{R}^{3}\right)\right), \quad \frac{3}{p}+\frac{2}{r} \leq 1,
$$

for $3<p \leq \infty$, then $v(x, t)$ is regular. After that there are further developments and refinements by many authors including $[20,22,19,31,33]$. In particular, Beirão da Veiga [3] obtained a regularity condition in terms of $\nabla v$, which is equivalent to the one in terms of the vorticity due to the Calderón-Zygmund inequality. This states that if the vorticity $\omega$ of the weak solution $v$ satisfies

$$
\omega \in L^{r}\left(0, T ; L^{p}\left(\mathbb{R}^{3}\right)\right), \quad \frac{3}{p}+\frac{2}{r} \leq 2,
$$

for $\frac{3}{2}<p \leq \infty$, then $v$ becomes regular. This condition was later improved in [9], requiring the same regularity condition only for the two components of the vorticity. On the other hand, Constantin and Fefferman discovered remarkable geometric structures of the vortex stretching term, the first one on the right hand side of (4), which leads to the following statements [13], see also [12]. Let $\xi(x, t)=\omega(x, t) /|\omega(x, t)|$ be the direction field of the vorticity, and let $\theta(x, y, t)$ be the angle between $\xi(x, t)$ and $\xi(x+y, t)$. If $\theta(x, y, t)$ satisfies

$$
|\sin \theta(x, y, t)| \leq C|y|
$$

in the region where $|\omega(x, t)|,|\omega(x+y, t)|>K$ for some large constant $K$, then the solution becomes regular. After that these geometric structures have been used by many authors, $[14,6,7,9,4,2,5,30]$. For the convenience in the later discussion of our main result we describe here the results in [4] and [2]. In [4] it is proved that if

$$
\begin{aligned}
|\sin \theta(x, y, t)| \leq g(x, t)|y|^{s}, & \text { for some function } g \in L^{r}\left(0, T ; L^{p}\left(\mathbb{R}^{3}\right)\right) \\
\text { with } & \frac{3}{p}+\frac{2}{r}=s-\frac{1}{2},
\end{aligned}
$$

for some $s \in[1 / 2,1], r \in\left[\frac{4}{2 s-1}, \infty\right]$ in the region where $|\omega(x, t)|,|\omega(x+y, t)|>K$ for some large constant $K$, then the solution becomes regular on $[0, T]$. We note here that for $p=r=\infty, s=1 / 2$ the condition (9) reduces to

$$
|\sin \theta(x, y, t)| \leq C|y|^{\frac{1}{2}}
$$


which shows a genuine improvement of [13]. In order to handle the case $s \in(0,1 / 2]$ in (9) the following is proved in [2]. If

$$
|\sin \theta(x, y, t)| \leq|y|^{s} \quad \text { and } \quad \omega \in L^{2}\left(0, T ; L^{p}\left(\mathbb{R}^{3}\right)\right), \quad \frac{3}{p}=s+1
$$

for some $s \in\left(0, \frac{1}{2}\right]$ in the region where $|\omega(x, t)|,|\omega(x+y, t)|>K$ for some large constant $K$, then the solution becomes regular on $[0, T]$. We observe here that there is an extra condition of suitable integrability of the vorticity besides the regularity condition of the direction. Before stating our main theorems below we note that all of the above regularity conditions for weak solutions can be viewed as a continuation principle for local in time strong solutions in $H^{s}\left(\mathbb{R}^{3}\right), s>5 / 2$. Our first theorem can be considered as the extension of the result in [3].

THEOREM 1. Let $\omega$ be the vorticity of a solution of $v$ for $(N S)_{\alpha}, 0<\alpha \leq 2$, satisfying

$$
\omega \in L^{r}\left(0, T ; L^{p}\left(\mathbb{R}^{3}\right)\right), \quad \text { with } \quad \frac{3}{p}+\frac{\alpha}{r} \leq \alpha,
$$

where $\frac{6}{\alpha}<p \leq \infty$. Then, there is no singularity up to $T$.

The full proof of the above theorem is in [6]. We just point out here that we use the BKM criterion [1] as the crucial continuation principle, and use the pointwise inequality for the fractional derivatives

$$
\left(\Lambda^{\alpha} f^{2}\right)(x) \leq 2 f(x)\left(\Lambda^{\alpha} f\right)(x)
$$

see [17], during our main part of the estimates.

REMARK 1. The above theorem says quantitatively that for the regularity of solutions with a weaker dissipative term we need higher integrability of the vorticity. For $\alpha=2$ (11) reduces to (8). For $p=\infty, r=1$ (11) reduces to the Beale-Kato-Majda condition in $[1]$.

REMARK 2. We observe that the system $(N S)_{\alpha}$ is invariant under scaling transform

$$
v(x, t) \mapsto v^{\lambda}(x, t)=\lambda^{\alpha-1} v\left(\lambda x, \lambda^{\alpha} t\right), \quad p(x, t) \mapsto p^{\lambda}(x, t)=\lambda^{2 \alpha-2} p\left(\lambda x, \lambda^{\alpha} t\right),
$$

which induces the scaling for the vorticity, $\omega(x, t) \mapsto \omega^{\lambda}(x, t)=\lambda^{\alpha} \omega\left(\lambda x, \lambda^{\alpha} t\right)$. For this scaling transform we have the norm invariance in the case of equality in (11) as follows

$$
\|\omega\|_{L^{r}\left(0, T ; L^{p}\left(\mathbb{R}^{3}\right)\right)}=\left\|\omega^{\lambda}\right\|_{L^{r}\left(0, \lambda^{\alpha} T ; L^{p}\left(\mathbb{R}^{3}\right)\right)}, \quad \text { if } \quad \frac{3}{p}+\frac{\alpha}{r}=\alpha .
$$

In this sense we can regard the condition (11) as optimal.

For the statement of our next result we introduce a function space. Given $0<s<1$, $1 \leq p \leq \infty, 1 \leq q \leq \infty$, the function space $\dot{\mathcal{F}}_{p, q}^{s}$ is defined by the seminorm

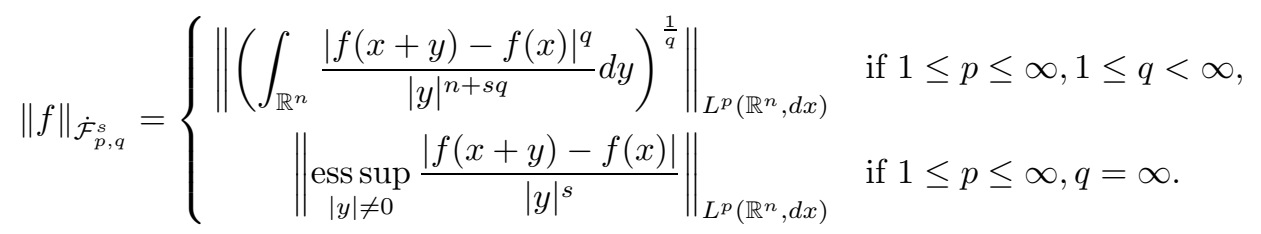


Observe that, in particular, $\dot{\mathcal{F}}_{\infty, \infty}^{s} \cong C^{s}$, the usual Hölder seminormed space. In order to compare this space with other more classical function spaces let us introduce the Banach space $\mathcal{F}_{p, q}^{s}$ by defining its norm

$$
\|f\|_{\mathcal{F}_{p, q}^{s}}=\|f\|_{L^{p}}+\|f\|_{\dot{\mathcal{F}}_{p, q}^{s}} .
$$

We note that for $0<s<1,2 \leq p<\infty$ and $q=2, \mathcal{F}_{p, 2}^{s} \cong L_{s}^{p}\left(\mathbb{R}^{n}\right)=(1-\Delta)^{-\frac{s}{2}} L^{p}\left(\mathbb{R}^{n}\right)$, the fractional order Sobolev space (or the Bessel potential space), see [34, p. 163]). If $\frac{n}{\min \{p, q\}}<s<1, n<p<\infty$ and $n<q \leq \infty$, then $\mathcal{F}_{p, q}^{s}$ coincides with the TriebelLizorkin space $F_{p, q}^{s}$, see [36, p. 101]). The following is our main Theorem.

TheOrem 2. Let $v(x, t)$ be a solution to $(N S)_{\alpha}$, and $\omega(x, t)=\operatorname{curl} v(x, t)$. Let $\xi(x, t)$ be its direction field $\xi(x, t)=\omega(x) /|\omega(x)|$ defined for $\omega(x, t) \neq 0$. Suppose there exist $s \in(0,1), q \in\left(\frac{3}{3-s}, \infty\right], p_{1} \in(1, \infty], p_{2} \in\left(1, \frac{3}{s}\right)$, satisfying $\frac{s}{3}<\frac{1}{p_{1}}+\frac{1}{p_{2}}<\frac{\alpha+s}{3}$, $\frac{1}{p_{2}}+\frac{1}{q}<1+\frac{s}{3}$ and $r_{1}, r_{2} \in[1, \infty]$ such that the following hold:

$$
\begin{gathered}
\xi(x, t) \in L^{r_{1}}\left(0, T ; \dot{\mathcal{F}}_{p_{1}, q}^{s}\right) \quad \text { and } \quad \omega(x, t) \\
\text { with } \left.\frac{3}{p_{1}}+\frac{3}{p_{2}}+\frac{\alpha}{r_{1}}+\frac{\alpha}{r_{2}} \leq \alpha+T ; L^{p_{2}}\left(\mathbb{R}^{3}\right)\right)
\end{gathered}
$$

Then, there is no singularity up to $T$.

The proof of the above theorem is also in [6]. We just mention here that we use special structure of the 'vortex stretching term', and apply Theorem 1 as the key ingredients of the proof.

REMARK 3. Intuitively, the above theorem says that assumption of higher regularity of the direction vector field compensates the weaker assumption on the integrability of the amplitude of vorticity field.

REMARK 4 . We consider the two special cases for $(N S)$ below ( $\alpha=2$ case). First, let $p_{2}=r_{2}=2$. Then, we know that the Leray-Hopf weak solution $\omega$ satisfies

$$
\int_{0}^{T}\|\omega(t)\|_{L^{2}}^{2} d t<\infty
$$

and the condition of the above theorem reduces to

$$
\xi(x, t) \in L^{r_{1}}\left(0, T ; \dot{\mathcal{F}}_{p_{1}, q}^{s}\right), \quad \frac{3}{p_{1}}+\frac{2}{r_{1}} \leq s-\frac{1}{2} .
$$

Comparing this with (9), we find the natural identification of the function $g(x, t)$ as the direction field $\xi(x, t)$. Moreover, since we allow any finite number $q$ in $\left(\frac{3}{3-s}, \infty\right]$, not necessarily infinity, the condition (13) is a generalization of (9).

Secondly, we observe that in the case $p_{1}=r_{1}=\infty$, and $s \in\left(0, \frac{1}{2}\right]$ the condition of the above theorem reduces to

$$
\xi \in \dot{\mathcal{F}}_{\infty, q}^{s}, \quad \omega \in L^{r_{2}}\left(0, T ; L^{p_{2}}\left(\mathbb{R}^{3}\right)\right) \quad \text { with } \quad \frac{3}{p_{2}}+\frac{2}{r_{2}} \leq s+2 .
$$

Since $\dot{\mathcal{F}}_{\infty, \infty}^{s} \cong C^{s}$, and $|\sin \theta(x, y, t)| \leq|\xi(x+y, t)-\xi(x, t)|$, we have

$$
|\sin \theta(x, y, t)| \leq C|y|^{s} \quad \text { if } \quad \xi \in L^{\infty}\left(0, T ; \dot{\mathcal{F}}_{\infty, \infty}^{s}\right) .
$$

Hence, we find that (10) is a special case for $q=\infty, r_{2}=2$ of (14). 
Finally, we note that Theorem 2 is recently localized by the author of this article and his collaborators, [10].

2. The Euler equations. In this section we are concerned with the following homogeneous incompressible Euler equations in $\mathbb{R}^{3}$ :

$$
\begin{gathered}
\frac{\partial v}{\partial t}+(v \cdot \nabla) v=-\nabla p, \\
\operatorname{div} v=0, \\
v(x, 0)=v_{0}(x) .
\end{gathered}
$$

The results in the previous section could not be straightforwardly extended to the inviscid case of the Euler equations, mainly due to the fact that the Riesz potential behaves badly for the $L^{\infty}$ estimates. We need a different type of potential function, and based on the $L^{\infty}$ estimate of this potential we extend the previous regularity criterion of the Navier-Stokes equations to the Euler equations, see [7] for a detailed proof.

THEOREM 3. Let $v(x, t)$ be the local classical solution to (15)-(17) with initial data $v_{0} \in H^{m}\left(\mathbb{R}^{3}\right), m>5 / 2$, and $\omega(x, t)=\operatorname{curl} v(x, t)$. Then, the solution can be continued up to $T<\infty$ as the classical solution, namely $v(t) \in C\left([0, T] ; H^{m}\left(\mathbb{R}^{3}\right)\right)$, if there exist $p, p^{\prime}, q, q^{\prime}, s, r_{1}, r_{2}, r_{3}$ satisfying the following conditions:

$$
\frac{1}{p}+\frac{1}{p^{\prime}}=1, \quad \frac{1}{q}+\frac{1}{q^{\prime}}=1,
$$

and

$$
\frac{1}{r_{1}}+\frac{p^{\prime}}{r_{2}}\left(1-\frac{s q^{\prime}}{3}\right)+\frac{1}{r_{3}}\left\{1-p^{\prime}\left(1-\frac{s q^{\prime}}{3}\right)\right\}=1
$$

with

$$
0<s<1, \quad 1 \leq \frac{3}{s q^{\prime}}<p \leq \infty, \quad 1 \leq q \leq \infty
$$

and

$$
r_{1} \in[1, \infty], r_{2} \in\left[p^{\prime}\left(1-\frac{s q^{\prime}}{3}\right), \infty\right], r_{3} \in\left[1-p^{\prime}\left(1-\frac{s q^{\prime}}{3}\right), \infty\right],
$$

such that for the direction field $\xi(x, t)$, and the magnitude of vorticity $|\omega(x, t)|$ the following hold:

$$
\int_{0}^{T}\|\xi(t)\|_{\dot{\mathcal{F}}_{\infty, q}^{s}}^{r_{1}} d t<\infty
$$

and

$$
\int_{0}^{T}\|\omega(t)\|_{L^{p q^{\prime}}}^{r_{2}} d t+\int_{0}^{T}\|\omega(t)\|_{L^{q^{\prime}}}^{r_{3}} d t<\infty .
$$

REMARK 5. Let us consider the special case of $p=\infty, q=1$. In this case the conditions (22)-(23) are satisfied if

$$
\begin{gathered}
\xi(x, t) \in L^{r_{1}}\left(0, T ; C^{s}\left(\mathbb{R}^{3}\right)\right), \\
\omega(x, t) \in L^{r_{2}}\left(0, T ; L^{\infty}\left(\mathbb{R}^{3}\right)\right) \cap L^{r_{3}}\left(0, T ; L^{\infty}\left(\mathbb{R}^{3}\right)\right) .
\end{gathered}
$$

with

$$
\frac{1}{r_{1}}+\frac{1}{r_{2}}\left(1-\frac{s}{3}\right)+\frac{s}{3 r_{3}}=1 .
$$


In order to understand these conditions more intuitively we formally pass to the limit $s \rightarrow 0$ in (24) and (26), and choose $r_{1}=\infty$ and $r_{2}=r_{3}=1$, then we find that the conditions $(24)-(25)$ reduce to the Beale-Kato-Majda's condition, since the condition $\xi(x, t) \in L^{\infty}\left(0, T ; C^{0}\left(\mathbb{R}^{3}\right)\right) \cong L^{\infty}\left((0, T) \times \mathbb{R}^{3}\right)$ is obviously satisfied due to the fact that $|\xi(x, t)| \equiv 1$.

REMARK 6. The other case of interest is $q^{\prime}=3 / s$, where $(22)-(23)$ are satisfied if

$$
\xi(x, t) \in L^{r_{1}}\left(0, T ; \dot{\mathcal{F}}_{\infty, \frac{3}{3-s}}^{s}\left(\mathbb{R}^{3}\right)\right), \quad|\omega(x, t)| \in L^{r_{2}}\left(0, T ; L^{\frac{3}{s}}\left(\mathbb{R}^{3}\right)\right),
$$

with $1 / r_{1}+1 / r_{2}=1$. The condition (27) shows explicitly the mutual compensation between the regularity of the direction field and the integrability of the vorticity magnitude in order to control regularity/singularity of solutions of the Euler equations.

3. The quasi-geostrophic equations. Here we are concerned with the regularity of the quasi-geostrophic equation with a dissipation term.

$$
\begin{gathered}
\frac{\partial \theta}{\partial t}+(v \cdot \nabla) \theta=-\kappa \Lambda^{\alpha} \theta \\
v(x, t)=-\nabla^{\perp}(-\Delta)^{-\frac{1}{2}} \theta=-\int_{\mathbb{R}^{3}} \frac{\nabla^{\perp} \theta(x+y, t)}{|y|} d y \\
\theta(x, 0)=\theta_{0}(x)
\end{gathered}
$$

where $\theta(x, t)$ is a scalar function representing temperature, $v(x, t)$ is the velocity field of the fluid, $\kappa \geq 0$ is the diffusion constant, $\Lambda^{\alpha}=(-\Delta)^{\frac{\alpha}{2}}$, and $\nabla^{\perp}=\left(-\partial_{x_{2}}, \partial_{x_{1}}\right)$. See e.g. $[15,12,27]$ for the instructive discussions and the physical and mathematical motivations for the study of (28)-(30), in particular for the inviscid case $\kappa=0$. For $\alpha>1$ the global regularity of solution of (28)-(30) is well-known (see [16]). On the other hand, for $0 \leq \alpha \leq 1$, the question of global regularity/finite time singularity is still a challenging open problem, see e.g. [11, 17, 18, 16, 37, 38, 39] for related studies. In particular, the critical dissipation $\alpha=1$ case has the similar features to the 3D Navier-Stokes equations, and could be considered as its model problem. In order to see the similarities to the 3D Navier-Stokes equations with fractional powers of Laplacian more apparently we apply the operation $\nabla^{\perp}$ to $(28)$ to obtain

$$
\frac{\partial \nabla^{\perp} \theta}{\partial t}+(v \cdot \nabla) \nabla^{\perp} \theta=\left(\nabla^{\perp} \theta \cdot \nabla\right) v-\kappa \Lambda^{\alpha} \nabla^{\perp} \theta
$$

Then, we observe $\nabla^{\perp} \theta$ has the role of vorticity, and (29) corresponds to the Biot-Savart law for the 3D Navier-Stokes equations. In this note we are concerned with sufficient conditions to guarantee regularity of solutions of the quasi-geostrophic equations, which have been studied by many authors for case of the 3D Navier-Stokes equations. To the author's knowledge the only regularity condition available in the literature for (28)-(30) is the following one obtained by Constantin, Majda and Tabak [15]:

$$
\limsup _{t \nearrow T}\|\theta(t)\|_{H^{m}}<\infty \text { if and only if } \int_{0}^{T}\left\|\nabla^{\perp} \theta(t)\right\|_{L^{\infty}} d t<\infty,
$$

where $m>2$, which holds for solutions of both viscous and inviscid $(\kappa=0)$ equations. Our first theorem in this section generalizes this as follows, the proof of which is in [8]. 
THEOREM 4. Let $\theta(x, t)$ be a solution of the quasi-geostrophic equation (28)-(30) with $\alpha \in(0,1], \kappa>0$, and its derivative, $\nabla^{\perp} \theta$ satisfy

$$
\nabla^{\perp} \theta \in L^{r}\left(0, T ; L^{p}\left(\mathbb{R}^{2}\right)\right) \quad \text { for some } p, r \text { with } \quad \frac{2}{p}+\frac{\alpha}{r} \leq \alpha, \quad \frac{2}{\alpha}<p<\infty
$$

then there is no singularity up to $T$.

REMARK 7 . We observe that $p=\infty, r=1$, corresponds to $(32)$ for any $\alpha \in(0,1]$.

REMARK 8 . We note that the system (28)-(29) has the symmetry under the scaling transform,

$$
\theta(x, t) \mapsto \theta^{\lambda}(x, t)=\lambda^{\alpha-1} \theta\left(\lambda x, \lambda^{\alpha} t\right) .
$$

Under this scaling transform we have the invariance of the norms

$$
\left\|\nabla^{\perp} \theta\right\|_{L^{r}\left(0, T ; L^{p}\left(\mathbb{R}^{2}\right)\right)}=\left\|\nabla^{\perp} \theta^{\lambda}\right\|_{L^{r}\left(0, \lambda^{\alpha} T ; L^{p}\left(\mathbb{R}^{2}\right)\right)} \quad \text { if } \quad \frac{2}{p}+\frac{\alpha}{r}=\alpha .
$$

In this sense the condition (33) is optimal.

Using theorem 4, following a similar argument to the proof of Theorem 2, we can prove the following, the detailed proof of which is in [8].

THEOREM 5. Let $\theta(x, t)$ be a solution of the dissipative quasi-geostrophic equations, (28)-(30) with $\kappa>0$. Let $\xi(x, t)$ be its direction field, $\xi(x, t)=\nabla^{\perp} \theta(x) /\left|\nabla^{\perp} \theta(x)\right|$ defined for $\nabla^{\perp} \theta(x, t) \neq 0$. Suppose there exists $s \in(0,1), q \in\left(\frac{2}{2-s}, \infty\right], p_{1} \in(1, \infty], p_{2} \in\left(1, \frac{2}{s}\right)$, satisfying $\frac{s}{2}<\frac{1}{p_{1}}+\frac{1}{p_{2}}<\frac{s+\alpha}{2}$, and $r_{1}, r_{2} \in[1, \infty]$ such that the following hold.

$$
\begin{gathered}
\xi(x, t) \in L^{r_{1}}\left(0, T ; \dot{\mathcal{F}}_{p_{1}, q}^{s}\right) \quad \text { and } \nabla^{\perp} \theta(x, t) \in L^{r_{2}}\left(0, T ; L^{p_{2}}\left(\mathbb{R}^{2}\right)\right) \\
\text { with } \quad \frac{2}{p_{1}}+\frac{2}{p_{2}}+\frac{\alpha}{r_{1}}+\frac{\alpha}{r_{2}} \leq \alpha+s
\end{gathered}
$$

Then, the solution $\theta(x, t)$ is regular up to $T$.

REMARK 9. The condition (34) describes quantitatively that by assuming regularity of the direction field $\xi(x, t)$ we can have regularity of solution with an assumption of lower integrability of $\left|\nabla^{\perp} \theta(x, t)\right|$ than required in Theorem 4 .

The following result could be considered as an inviscid extension of Theorem 5 , the proof of which is similar to that of Theorem 3, cf. [7].

THEOREM 6. Let $\theta(x, t)$ be the local classical solution to $(Q G)$ with initial data $\theta_{0} \in$ $H^{m}\left(\mathbb{R}^{2}\right), m>3 / 2$. Let us set $\xi(x, t)=\nabla^{\perp} \theta(x, t) /\left|\nabla^{\perp} \theta(x, t)\right|$. Then, the solution can be continued up to $T<\infty$ as the classical solution, namely $\theta(t) \in C\left([0, T] ; H^{m}\left(\mathbb{R}^{2}\right)\right)$, if there exist parameters $p, p^{\prime}, q, q^{\prime}, s, r_{1}, r_{2}, r_{3}$ satisfying the following conditions:

$$
\frac{1}{p}+\frac{1}{p^{\prime}}=1, \quad \frac{1}{q}+\frac{1}{q^{\prime}}=1
$$

and

$$
\frac{1}{r_{1}}+\frac{p^{\prime}}{r_{2}}\left(1-\frac{s q^{\prime}}{2}\right)+\frac{1}{r_{3}}\left\{1-p^{\prime}\left(1-\frac{s q^{\prime}}{2}\right)\right\}=1
$$

with

$$
0<s<1, \quad 1 \leq \frac{2}{s q^{\prime}}<p \leq \infty, \quad 1 \leq q \leq \infty
$$


and

$$
r_{1} \in[1, \infty], r_{2} \in\left[p^{\prime}\left(1-\frac{s q^{\prime}}{2}\right), \infty\right], r_{3} \in\left[1-p^{\prime}\left(1-\frac{s q^{\prime}}{2}\right), \infty\right]
$$

such that the following hold:

$$
\int_{0}^{T}\|\xi(t)\|_{\mathcal{\mathcal { F }}_{\infty, q}}^{r_{1}} d t<\infty
$$

and

$$
\int_{0}^{T}\left\|\nabla^{\perp} \theta(t)\right\|_{L^{p q^{\prime}}}^{r_{2}} d t+\int_{0}^{T}\left\|\nabla^{\perp} \theta(t)\right\|_{L^{q^{\prime}}}^{r_{3}} d t<\infty .
$$

REMARK 10. In the case of $p=\infty, q=1$ the conditions (39)-(40) are satisfied if

$$
\begin{gathered}
\xi(x, t) \in L^{r_{1}}\left(0, T ; C^{s}\left(\mathbb{R}^{2}\right)\right), \\
\left|\nabla^{\perp} \theta(x, t)\right| \in L^{r_{2}}\left(0, T ; L^{\infty}\left(\mathbb{R}^{2}\right)\right) \cap L^{r_{3}}\left(0, T ; L^{\infty}\left(\mathbb{R}^{2}\right)\right)
\end{gathered}
$$

with

$$
\frac{1}{r_{1}}+\frac{1}{r_{2}}\left(1-\frac{s}{2}\right)+\frac{s}{2 r_{3}}=1
$$

Here also, for heuristic understanding, formally passing to the limit $s \rightarrow 0$, and choosing $r_{1}=\infty, r_{2}=r_{3}=1$, we find that the conditions (41)-(42) are satisfied if the ConstantinMajda-Tabak condition [15] is, since the condition

$$
\xi(x, t) \in L^{\infty}\left(0, T ; C^{0}\left(\mathbb{R}^{2}\right)\right) \cong L^{\infty}\left((0, T) \times \mathbb{R}^{2}\right)
$$

is automatically satisfied. The other is the case $q^{\prime}=2 / s$, where (39)-(40) are satisfied if

$$
\xi(x, t) \in L^{r_{1}}\left(0, T ; \dot{\mathcal{F}}_{\infty, \frac{2}{2-s}}^{s}\left(\mathbb{R}^{2}\right)\right), \quad\left|\nabla^{\perp} \theta(x, t)\right| \in L^{r_{2}}\left(0, T ; L^{\frac{2}{s}}\left(\mathbb{R}^{2}\right)\right)
$$

with $1 / r_{1}+1 / r_{2}=1$, which shows mutual compensation of the regularity of the direction field $\xi(x, t)$ and the integrability of the magnitude of gradient $\left|\nabla^{\perp} \theta(x, t)\right|$ to obtain smoothness of $\theta(x, t)$.

\section{References}

[1] J. T. Beale, T. Kato and A. Majda, Remarks on the breakdown of smooth solutions for the 3-D Euler equations, Comm. Math. Phys. 94 (1984), 61-66.

[2] H. Beirão da Veiga, Vorticity and smoothness in incompressible viscous flows, in: Wave Phenomena and Asymptotc Analysis, RIMS, Kokyuroku 1315 (2003), 37-45.

[3] H. Beirão da Veiga, Concerning the regularity problem for the solutions of the NavierStokes equations, C. R. Acad. Sci. Paris Sér. I Math. 321 (1995), 405-408.

[4] H. Beirão da Veiga, Existence and asymptotic behavior for strong solutions of the NavierStokes equations in the whole space, Indiana Univ. Math. J. 36 (1987), 149-166.

[5] H. Beirão da Veiga and L. C. Berselli, On the regularizing effect of the vorticity direction in incompressible viscous flows, Diff. Int. Eqns, 15, (2002), 345-356.

[6] D. Chae, On the regularity conditions for the Navier-Stokes and related equations, Revista Mat. Iberoamericana, to appear (2006).

[7] D. Chae, On the continuation principles for the Euler equations and the quasi-geostrophic equation, J. Diff. Eq. 227 (2006), 640-651. 
[8] D. Chae, On the regularity conditions for the dissipative quasi-geostrophic equations, SIAM J. Math. Anal. 37 (2006), 1649-1656.

[9] D. Chae and H.-J. Choe, Regularity of solutions to the Navier-Stokes equations, Electronic J. Diff. Eq. (1999), 1-7.

[10] D. Chae, K. Kang and J. Lee, On the interior regularity of suitable weak solutions to the Navier-Stokes equations, Comm. Partial Diff. Eqns, to appear (2006).

[11] D. Chae and J. Lee, Global well-posedness in the super critical dissipative quasi-geostrophic equations, Comm. Math. Phys. 233 (2003), 297-311.

[12] P. Constantin, Geometric statistics in turbulence, SIAM Rev. 36 (1994), 73-98.

[13] P. Constantin and C. Fefferman, Direction of vorticity and the problem of global regularity for the Navier-Stokes equations, Indiana Univ. Math. J. 42 (1993), 775-789.

[14] P. Constantin, C. Fefferman and A. Majda, Geometric constraints on potential singularity solutions for the 3D Euler equations, Comm. Partial Diff. Eq. 21 (1996), 559-571.

[15] P. Constantin, A. Majda and E. Tabak, Formation of strong fronts in the 2-D quasigeostrophic thermal active scalar, Nonlinearity 7 (1994), 1459-1533.

[16] P. Constantin, D. Córdoba and J. Wu, On the critical dissipative quasi-geostrophic equation, Indiana Univ. Math. J. 50 (2001), 97-107.

[17] A. Córdoba and D. Córdoba, A maximum principle applied to quasi-geostrophic equations, Comm. Math. Phys. 249 (2004), 511-528.

[18] D. Córdoba and C. Fefferman, Growth of solutions for $Q G$ and $2 D$ Euler equations, J. Amer. Math. Soc. 15 (2002), 665-670.

[19] L. Escauriaza, G. Seregin and V. Sverak, $L^{3, \infty}$-solutions of Navier-Stokes equations and backward uniqueness, Russian Math. Surveys 58 (2003), no. 2, 211-250.

[20] E. Fabes, B. Jones and N. Rivière, The initial value problem for the Navier-Stokes equations with data in $L^{p}$, Arch. Rat. Mech. Anal. 45 (1972), 222-248.

[21] Z. Grujić and A. Ruzmaikina, Interpolation between algebraic and geometric conditions for smoothness of the vorticity in the 3D NSE, Indiana Univ. Math. J. 53 (2004), 1073-1080.

[22] H. Kozono and Y. Taniuchi, Bilinear estimates in BMO and Navier-Stokes equations, Math. Z, 235 (2000), 173-194.

[23] O. A. Ladyzhenskaya, On the uniqueness and smoothness of generalized solutions of the Navier-Stokes equations, Zapiski N. Sem. LOMI 5 (1967), 169-185.

[24] O. A. Ladyzhenskaya, The Mathematical Theory of Viscous Incompressible Flow, Gordon and Breach, 1969.

[25] J. Leray, Essai sur le mouvement d'un fluide visqueux emplissant l'espace, Acta Math. 63 (1934), 193-248.

[26] J.-L. Lions, Quelques Méthodes de Résolution des Problèmes aux Limites Non Linéaires, Dunod, Paris, 1969.

[27] A. Majda and A. Bertozzi, Vorticity and Incompressible Flow, Cambridge Univ. Press, 2002 .

[28] G. Prodi, Un teorema di unicità per le equazioni di Navier-Stokes, Ann. Mat. Pura Appl. 48 (1959), 173-182.

[29] T. Ohyama, Interior regularity of weak solutions to the Navier-Stokes equation, Proc. Japan Acad. 36 (1960), 273-277.

[30] A. Ruzmaikina and Z. Grujić, On depletion of the vortex-stretching term in the 3D NavierStokes equations, Comm. Math. Phys. 247 (2004), 601-611. 
[31] G. Seregin and V. Sverak, The Navier-Stokes equations and backward uniqueness, in: Nonlinear Problems in Mathematical Physics and Related Topics, II, Int. Math. Ser. (N.Y.), 2, Kluwer/Plenum, New York, 2002, 353-366.

[32] J. Serrin, On the interior regularity of weak solutions of the Navier-Stokes equations, Arch. Rat. Mech. Anal. 9 (1962), 187-191.

[33] H. Sohr, A regularity class for the Navier-Stokes equations in Lorentz spaces, J. Evol. Eq. 1 (2001), 441-467.

[34] E. M. Stein, Singular Integrals and Differentiability Properties of Functions, Princeton Univ. Press, Princeton, NJ, 1970.

[35] R. Temam, Navier-Stokes Equations, 3rd ed., North-Holland, 1984.

[36] H. Triebel, Theory of Function Spaces, Birkhäuser Verlag, Boston, 1983.

[37] J. Wu, The quasi-geostrophic equations and its two regularizations, Comm. Partial Diff. Eq. 27 (2002), 1161-1181.

[38] J. Wu, Dissipative quasi-geostrophic equations with $L^{p}$ data, Electronic J. Diff. Eq. 56 (2001), 1-13.

[39] J. Wu, Inviscid limits and regularity estimates for the solutions of the 2-D dissipative quasi-geostrophic equations, Indiana Univ. Math. J. 46 (1997), 1113-1124. 